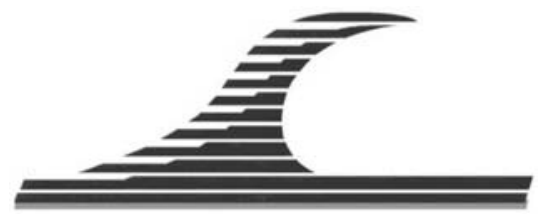

Revue Paralia, Volume 4 (2011) pp 8.1-8.11

Mots-clés : Sédiment de dragage, Liant hydraulique,

Correcteur granulométrique, Comportement physico-

mécanique, Microstructure

(C) Editions Paralia CFL

\title{
Influence de l'ajout d'un correcteur granulométrique sur les propriétés des sédiments traités aux liants hydrauliques
}

\author{
Mohamed BOUTOUIL ${ }^{1}$, Lucile SAUSSAYE ${ }^{1}$
}

1. ESITC Caen, Laboratoire de Recherche sur les Matériaux et la Construction (LRMC), 1 rue Pierre et Marie Curie, 14610 Epron, France.

dir.recherche@esitc-caen.fr, ecr.geotech@esitc-caen.fr

\section{Résumé :}

Dans la perspective de valorisation des sédiments marins dans des applications géotechniques routières, la présente étude concerne l'amélioration des propriétés physico-mécaniques des sédiments traités aux liants hydrauliques par ajout de correcteurs granulométriques.

Deux sables de caractéristiques physiques différentes, concassé et roulé, ont été incorporés à un sédiment fin non contaminé lors de son traitement au ciment. Les résultats de caractérisation mécanique montrent une amélioration de la résistance à la compression simple d'un facteur de plus de $40 \%$ après utilisation du sable concassé et 28 jours de cure normale. Aucune différence notable n’a été observée sur la résistance à la compression diamétrale entre les deux sables. Les analyses granulométriques et microstructurales au microscope électronique à balayage montrent une évolution continue de la microstructure dans le temps et permettent de mieux comprendre l'agencement des différents constituants de la nouvelle matrice.

Soumis le 9 décembre 2010, accepté le 21 octobre 2011, en ligne le 5 décembre 2011.

La seule version examinée est celle écrite en français. La ou les autres versions n'étant pas examinées par le comité de rédaction de la revue, sont donc publiées sous l'entière responsabilité du ou des auteurs.

A TRANSLATED VERSION IN ENGLISH IS AVAILABLE ONLINE

Pour citer cet article :

BOUTOUIL M., SAUSSAYE L. (2011). Influence de l'ajout d'un correcteur granulométrique sur les propriétés des sédiments traités aux liants hydrauliques. Revue Paralia, Vol. 4, pp 8.1-8.11.

DOI:10.5150/revue-paralia.2011.008

(disponible en ligne - http://www.paralia.fr - available online) 


\section{Introduction}

Au cours des deux dernières décennies, les autorités portuaires ont pris conscience que la gestion des matériaux de dragage d'entretien et/ou d'aménagement nécessite des solutions alternatives aux solutions traditionnelles de mise en dépôt à terre ou en mer. En effet ces dernières montrent leurs limites face à l'évolution de réglementations environnementales.

Les recherches menées en France (BOUTOUIL, 1998 ; REY, 1999 ; COLIN, 2003 ; DUBOIS, 2006 ; REKIK, 2007 ; DUAN, 2008) s'orientent de plus en plus vers la valorisation par utilisation des matériaux de dragage en application génie civil et vers l’intégration de la valorisation directement dans les projets de gestion des sédiments dragage (DAMIDOT et al., 2006 ; GROSDEMANGE et al., 2008).

Actuellement, parmi les techniques de valorisation diverses, la technique de solidification/stabilisation à base de liants hydrauliques possède une application potentielle reconnue, notamment grâce à la perspective de réutilisation des matériaux traités. Ses objectifs fondamentaux sont de réduire l'impact chimique négatif d'un déchet sur l'environnement tout en assurant l'obtention d'un produit ayant une intégrité structurelle (BOUTOUIL \& LEVACHER, 2000). Cependant, l'application de ce type de traitement sur des sédiments fins, caractérisés par une importante surface spécifique, engendre dans le matériau solidifié une porosité élevée, supérieure à 50\% (BOUTOUIL \& LEVACHER, 2001 ; REKIK \& BOUTOUIL, 2009). La résistance mécanique à long terme reste ainsi relativement faible.

Les études récentes sur la valorisation des sédiments de dragage incluent la possibilité d'utiliser un correcteur granulométrique (COLIN, 2003; SANNIER, 2008) en complément du traitement aux liants hydrauliques. Cet apport consiste à améliorer le squelette granulaire du matériau solidifié, répondant à une meilleure répartition granulométrique et permet de réduire les vides pour obtenir une compressibilité plus faible et une résistance mécanique plus élevée. Il atténue les paramètres de nature tels que l'argilosité, la teneur en matière organique (M.O.) et la teneur en eau initiale et il influe sur la mise en œuvre du matériau (LEVACHER et al., 2006).

Dans cette étude, le traitement à base de ciment CEMII/B-M (S-LL) $32.5 \mathrm{R}$ et de différents sables est appliqué selon différentes formulations à un sédiment fin non contaminé du port de Ouistreham (Calvados, France). L'influence de l'ajout de sable en tant que correcteur granulométrique est étudiée à travers les résistances mécaniques et l'évolution de la microstructure (granulométrie et observations au microscope électronique à balayage (MEB)).

\section{Matériaux et méthodes d'essais}

Le sédiment de dragage du port de Ouistreham est caractérisé par une granulométrie fine, avec 93\% de passant à $80 \mu \mathrm{m}$ (XP P 94-041) dont 25\% d'argiles (NF P 94-057). La valeur au bleu (NF P 94-068) atteint 3,5 g pour 100 g de sédiment sec. La teneur 
initiale en matières organiques, non modifiée au cours de l'étude, est de 7\% (XP P 94047). La teneur en eau du sédiment à l'extraction était de 127\% (NF P 94-050). Après trois années de stockage et de manipulations successives des bidons hermétiques (i.e. ouvertures et fermetures pour prélèvement de sédiment), la teneur en eau atteint $90 \%$ au moment de la réalisation de l'étude. Cette valeur est retenue comme étant la teneur en eau initiale du sédiment avant traitement, sans abaissement préalable.

Le liant hydraulique utilisé pour la technique de solidification / stabilisation est un ciment CEMII/B-M (S-LL) $32.5 \mathrm{R}$ caractérisé par une classe vraie à 28 jours de 46,2 MPa. Le dosage retenu, fixé à $10 \%$ par rapport à la masse humide initiale du sédiment, est pris en référence à l'étude de REKIK (2007). L’équivalent du dosage en ciment par rapport à la masse sèche du sédiment est de $19 \%$. Les valeurs de teneur en eau et de masse volumique sèche à l'optimum Proctor du sédiment traité sont respectivement de $38 \%$ et $1,11 \mathrm{t} / \mathrm{m}^{3}$ (REKIK, 2007).

Les effets de deux correcteurs granulométriques distincts sont recherchés pour des apports de 35, 40 et 45\% par rapport à la masse des constituants secs, sédiment et ciment. Ces trois dosages sont intermédiaires aux dosages de 30 et $50 \%$ sélectionnés par COLIN (2003).

Le premier est un sable roulé siliceux de granularité $0 / 4 \mathrm{~mm}$, le second, un grès feldspathique rouge concassé de classe $0 / 4$.

Ils diffèrent par leur nature, concassée et roulée, leur minéralogie et leur répartition granulométrique (voir figure 1).

Le sable roulé est caractérisé par un coefficient d'uniformité $\left(\mathrm{C}_{\mathrm{U}}\right)$ de 2,47 et par coefficient de courbure $\left(\mathrm{C}_{\mathrm{C}}\right)$ de 0,95 , caractéristiques d'un sable mal gradué. Son module de finesse (MF) est de 2,1.

Le sable concassé présente un $C_{U}$ de 50 et un $C_{C}$ de 0,78 , caractéristiques d'un sable bien gradué. Son MF est de 3,2. Ainsi, la courbe granulométrique du sable concassé s'étale sur un plus large spectre que celle du sable roulé.

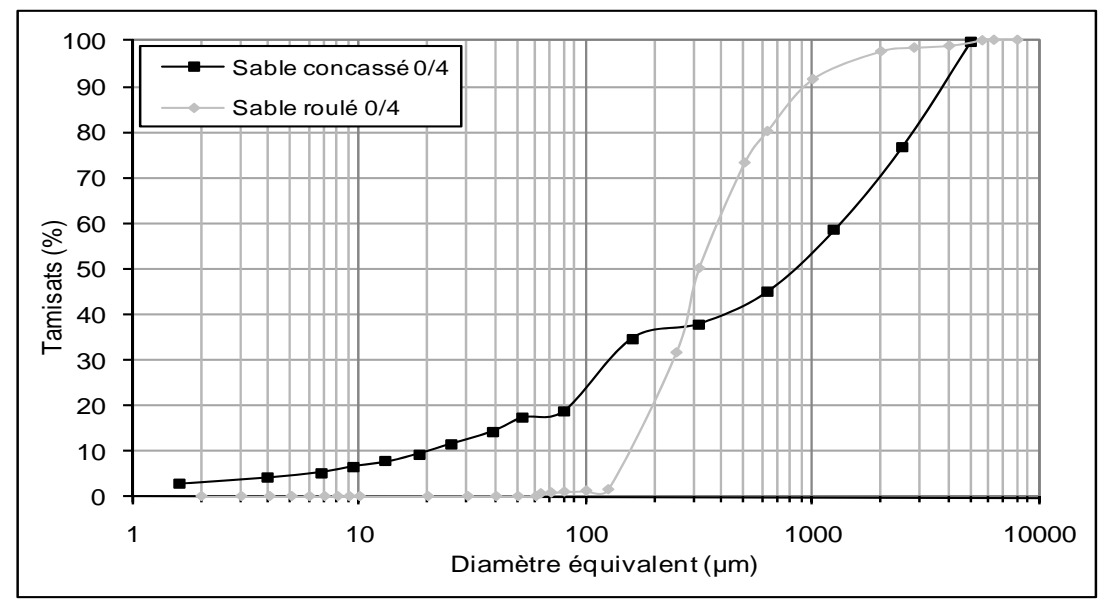

Figure 1. Courbes granulométriques des sables correcteurs utilisés. 
Les éprouvettes cylindriques $5 \times 10 \mathrm{~cm}$ et $5 \times 5 \mathrm{~cm}$ de matériau composite sont confectionnées à partir d'un mélange réalisé en incorporant successivement au sédiment, à sa teneur en eau initiale, le correcteur granulométrique puis le liant.

Les dimensions correspondent aux prescriptions du Guide technique de Traitement des Sols (GTS) (SETRA-LCPC, 2000).

Les calculs sont effectués pour s'approcher de la masse volumique sèche optimale de $1,11 \mathrm{t} \mathrm{m}^{-3}$ déterminée par REKIK (2007) sur le sédiment traité avec $10 \%$ de ciment. Cependant l'utilisation du sédiment à sa teneur en eau initiale, sans déshydratation préalable, empêche d'atteindre cet optimum. Les éprouvettes sont compactées manuellement en trois couches par une succession de 25 coups sur la paillasse d'essai pour atteindre les hauteurs de 5 ou $10 \mathrm{~cm}$ requises.

Trois éprouvettes sont confectionnées pour chaque formulation relative à chaque dosage de l'apport en sable. Elles sont conservées dans les conditions d'une cure normale, en moule hermétique à une température de $20 \pm 2{ }^{\circ} \mathrm{C}$, ou à l'air libre, et sont testées à 7 et 28 jours. Dans cette étude, sont présentés les résultats de caractérisation mécanique du matériau solidifié en compression simple (NF P98-232-1) et en compression diamétrale (NF P98-232-3) à une échéance de 28 jours.

Les analyses microstructurales (analyse granulométrique NF P94-056, sédimentométrie NF P94-057 et observations au MEB de type JEOL JSM 5300 LV en mode "électrons secondaires") ont été effectuées sur des échantillons issus d’éprouvettes soumises aux essais de résistance puis séchés à l'étuve à $50^{\circ} \mathrm{C}$.

\section{Résultats et analyses}

\subsection{Résistances mécaniques}

\subsubsection{Influence du dosage en sable correcteur}

Les essais de résistance à la compression simple $\left(\mathrm{R}_{\mathrm{c}}\right)$ réalisés à différentes échéances permettent de suivre la solidification du matériau traité. Les résultats des essais à 28 jours sur des éprouvettes conservées en cure normale sont présentés sur la figure 2. Dans l'optique d'une valorisation en construction routière, le GTS (SETRA-LCPC, 2000) préconise une valeur de $\mathrm{R}_{\mathrm{c}}$ de $1 \mathrm{MPa}$.

L'ajout de sable en tant que correcteur granulométrique permet d'améliorer la résistance à la compression simple. Quel que soit le type de sable, la résistance augmente par rapport à celle du sédiment traité sans apport i.e. échantillon témoin. Un ajout de sable dans l'intervalle des dosages étudiés conduit à un accroissement des performances. Pour un dosage de $45 \%$, on observe un doublement de celles-ci. 


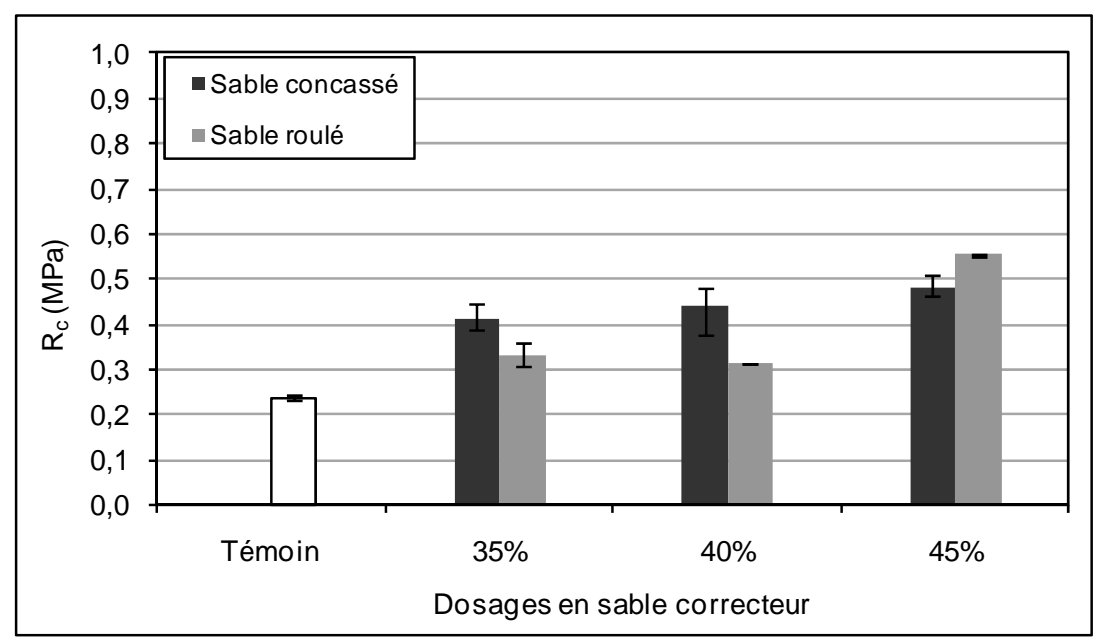

Figure 2. Résistances à la compression simple à 28 jours (moyennes et écarts-type) $d u$ sédiment traité avec 10\% de ciment, sans et avec 35, 40 et 45\% de sable correcteur, conservé en cure normale.

Le matériau intégrant le sable concassé développe des résistances voisines sur l'intervalle des dosages étudiés. La valeur moyenne des $R_{c}$ obtenues est de 0,44 MPa. Pour l'intervalle considéré, il existe une légère augmentation des performances avec l'augmentation du dosage en sable correcteur.

Le matériau à base de sable roulé conduit à une valeur de $\mathrm{R}_{\mathrm{c}}$ moyenne de 0,32 $\mathrm{MPa}$ pour les deux premiers dosages. Pour le dosage de $45 \%$, on obtient une valeur nettement plus élevée, de 0,55 MPa.

Les résultats des essais de résistance à la compression diamétrale $\mathrm{R}_{\mathrm{tb}}$, réalisés à 28 jours sur des éprouvettes conservées en cure normale, sont donnés à la figure 3. Ils viennent confirmer que l'apport d'un sable correcteur permet d'améliorer sensiblement les performances mécaniques du sédiment traité.

A 28 jours, quel que soit le dosage en sable correcteur, la valeur moyenne des $\mathrm{R}_{\mathrm{tb}}$ est de 0,10 MPa. Sans apport elle n'est que de 0,08 MPa. Pour un matériau à base de sable roulé dosé à $45 \%$, la $\mathrm{R}_{\mathrm{tb}}$ atteint $0,14 \mathrm{MPa}$, valeur la plus élevée. L'exigence fixée par le GTS (SETRA-LCPC, 2000) pour une réutilisation du matériau en couche de forme est une valeur de $\mathrm{R}_{\mathrm{tb}}$ de 0,25 MPa. 


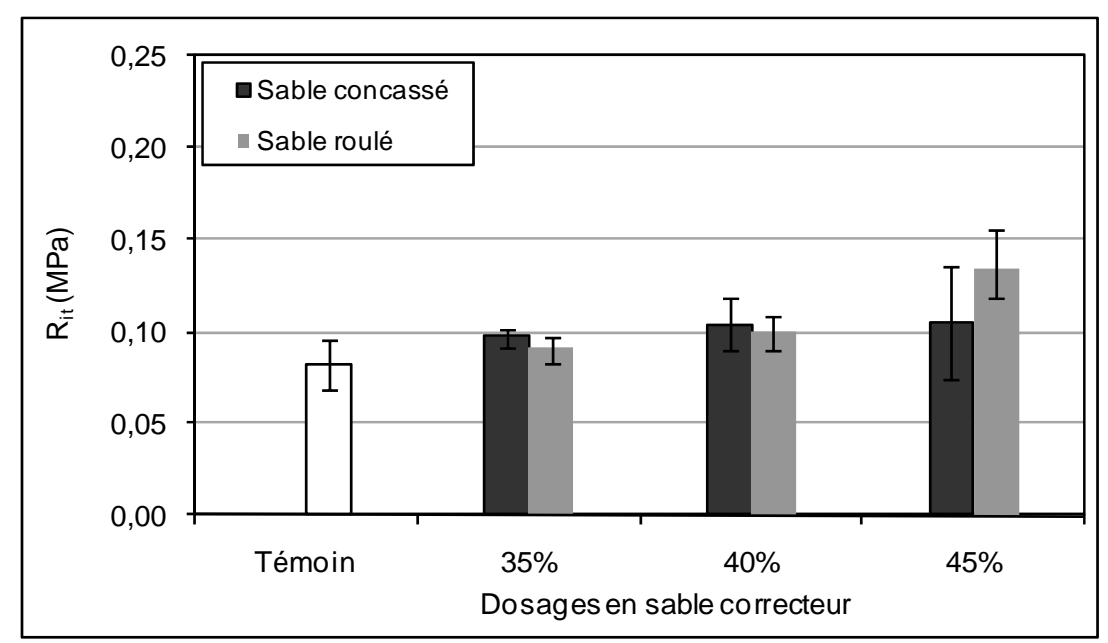

Figure 3. Résistances à la compression diamétrale à 28 jours (moyennes et écarts-type) du sédiment traité avec 10\% de ciment, sans et avec 35, 40 et 45\% de sable correcteur, conservé en cure normale.

\subsubsection{Influence de la nature granulométrique du correcteur}

Les résultats des essais de résistance à la compression simple, exposés à la figure 2, montrent que les performances développées sont moindres qu'avec l'apport de grès concassé.

Pour évaluer l'influence de la nature et de la répartition granulométrique des sables correcteurs sur les résistances à la compression simple, un troisième sable correcteur est proposé. Il s’agit du même sable concassé, modifié pour avoir la même granulométrie que celle du sable roulé donc le même $C_{U}$. Les résultats obtenus en compression simple après ajout de ce sable correcteur modifié au sédiment traité sont présentés sur la figure 4. Dans ce cas, les éprouvettes ont été conservées à l’air libre durant 28 jours avant d'être testées.

L’évolution apportée par un ajout de sable concassé modifié au sédiment de Ouistreham est marquée par une amélioration des résistances à la compression simple par rapport au témoin sans sable correcteur.

Ces performances sont aussi améliorées par rapport à celles du sédiment traité avec le sable roulé. La nature du sable est donc un facteur d'influence: le sable concassé favorise le développement des résistances mécaniques.

Néanmoins cette amélioration demeure plus faible que celle apportée par le sable concassé dans sa granulométrie originelle.

Il est ainsi démontré que la répartition granulométrique du sable roulé n’est pas la plus favorable au développement des résistances mécaniques au sein du matériau solidifié. L’incorporation d'un sable correcteur de granulométrie serrée au mélange conduirait à un empilement moins dense qu'avec un sable correcteur de granulométrie étalée. 


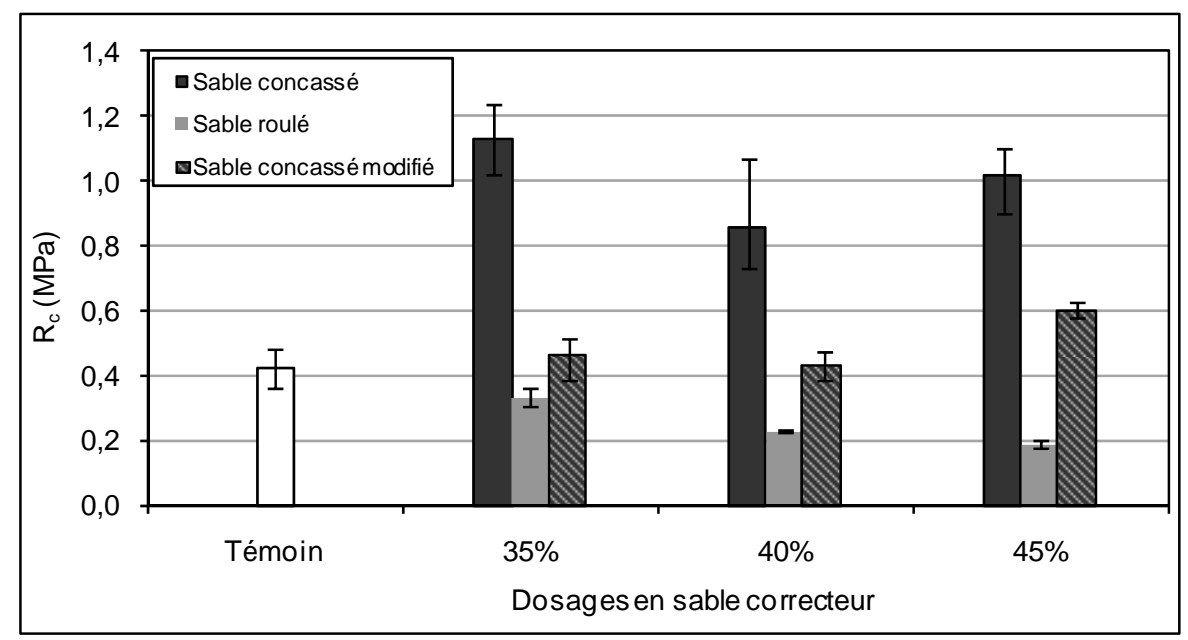

Figure 4. Résistances à la compression simple à 28 jours (moyennes et écarts-type) $d u$ sédiment traité avec 10\% de ciment, sans et avec 35, 40 et 45\% de sable correcteur, conservé à l'air libre.

La dispersion des mesures pour un matériau solidifié intégrant le sable concassé dans sa granulométrie initiale ne permet pas de conclure à un dosage préférentiel. Cette observation est valable quel que soit le mode de cure (voir les figures 2 et 4 ). Les incertitudes mesurées s'expliquent par une prise d'échantillon aléatoire qui ne garantit pas nécessairement la représentation de la totalité du spectre granulaire.

\subsection{Structure des sédiments solidifiés avec un sable correcteur}

\subsubsection{Analyse granulométrique des sédiments solidifiés}

Une analyse granulométrique des matériaux solidifiés a été effectuée afin de constater les modifications structurales. Les résultats ne sont mentionnés qu’à titre indicatif pour permettre de mieux cerner l'évolution générée par l'apport conjoint de sable correcteur et de liant hydraulique.

Après les essais de résistance, les éprouvettes sont séchées à l'étuve à $50{ }^{\circ} \mathrm{C}$, puis broyées manuellement au pilon dans un mortier en porcelaine. La figure 5 présente l'analyse granulométrique par sédimentométrie de matériaux solidifiés avec $45 \%$ de sable correcteur. Elle fait apparaître une granulométrie plus grossière par rapport au sédiment naturel et au sédiment traité sans apport de correcteur (témoin).

La complémentarité des deux traitements, liant et sable correcteur, se traduit par une augmentation de la taille des grains. La dilution des paramètres de nature, tels que les teneurs en eau, en matières organiques ou en argiles, associée à l'apport d'un nouvel élément de structure est immédiate ; le processus de floculation est suivi d'une phase de cimentation. 
La fraction inférieure à $2 \mu \mathrm{m}$, correspondant aux argiles, diminue, affectée par les deux processus cités.

La fraction à $80 \mu \mathrm{m}$ est elle aussi fortement réduite. Néanmoins les modifications apportées par le correcteur par rapport au témoin traité uniquement au ciment sont négligeables voire nulles.

Ainsi, comme il a été vu précédemment, une répartition granulométrique plus uniforme et plus étalée du sable correcteur semble pouvoir améliorer la compacité du squelette granulaire, les faibles différences de distributions granulométriques entre les matériaux solidifiés intégrant deux sables correcteurs distincts ne suffisent pas à expliquer l’accroissement des résistances mécaniques.

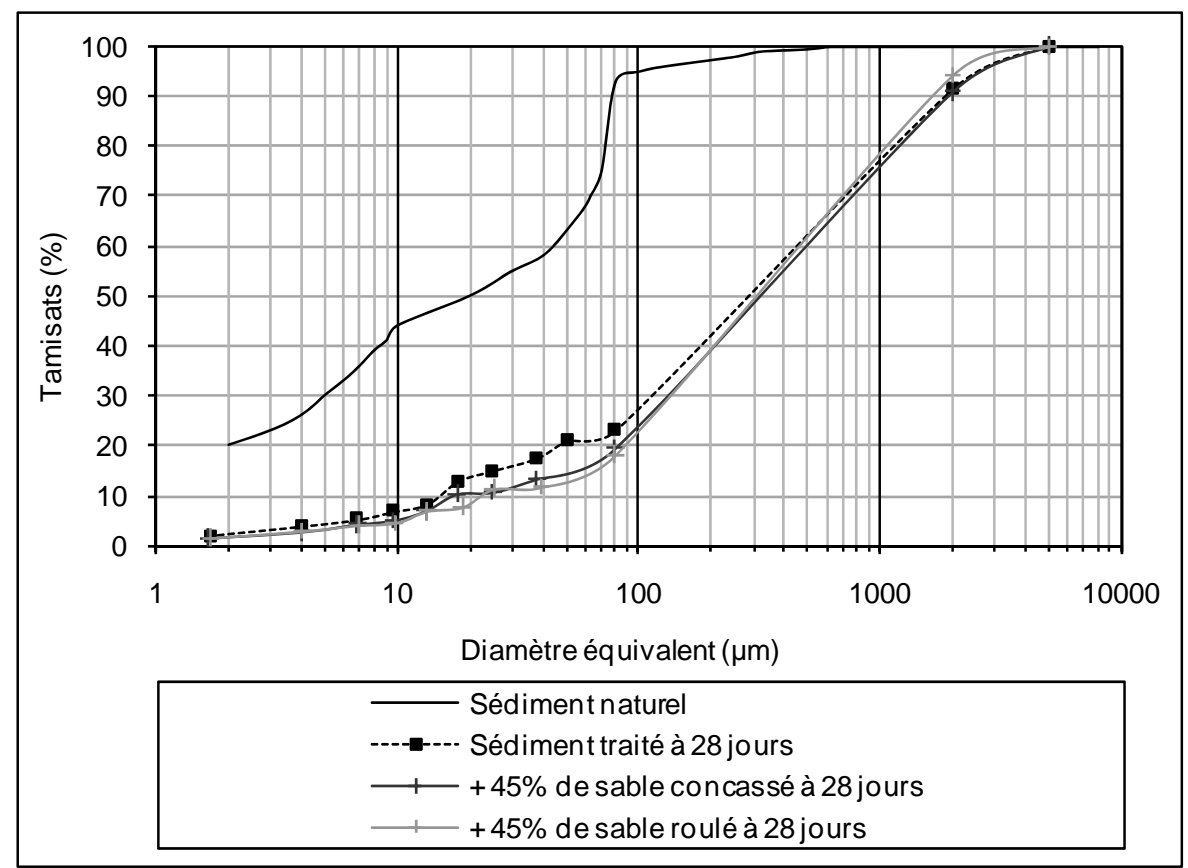

Figure 5. Analyse granulométrique à 28 jours du sédiment naturel et traité avec $10 \%$ de ciment, sans et avec $45 \%$ de sable correcteur.

\subsubsection{Observations au microscope électronique à balayage}

Les analyses d'images MEB permettent de mettre en avant l'évolution de la microstructure du sédiment traité et les interactions entre les différents constituants du mélange.

A 7 jours, les liens entre les éléments du squelette granulaire et la pâte de ciment sont superficiels. Les silicates de calcium hydratés (CSH), produits d'hydratation des silicates tricalciques du ciment, sont présents autour des grains du sédiment et du sable, améliorant la cohésion du mélange. L'évolution de la structure tend ensuite, jusqu'à 28 jours, vers une augmentation de la quantité de CSH et un renforcement de la structure. 
Influence de l'ajout d'un correcteur granulométrique sur les propriétés des sédiments traités aux liants hydrauliques : 8.9

En effet, à 28 jours, les clichés de la figure 6, pris avec un grossissement $\times 200$, montrent que les CSH sont les produits de l'hydratation du ciment majoritairement présents. Les grains de sédiments et de sable sont peu visibles, difficiles à discerner.

A 28 jours encore, les clichés de la figure 6 pris à un grossissement $\times 1500$, permettent de mieux comprendre ce qu'il se passe à l'interface. Les grains de sable concassé observés lors de l'analyse microscopique sont caractérisés par leur forme anguleuse. Ils sont englobés dans la matrice cimentaire et souvent constellés d'hydrates de ciment (les points d'emprise de la pâte de ciment sur le granulat sont matérialisés par les cercles). Pour les formulations intégrant un sable roulé, le contact est moins marqué, plus ponctuel.
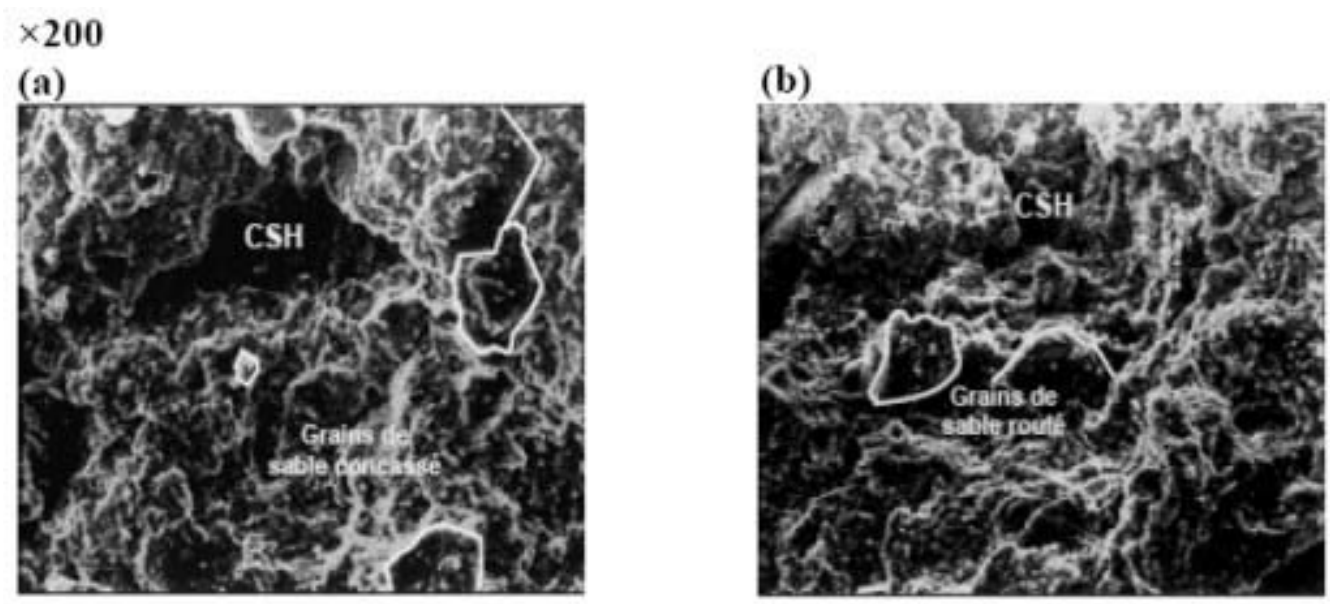

\section{$\times 1500$}

(a')

(b')
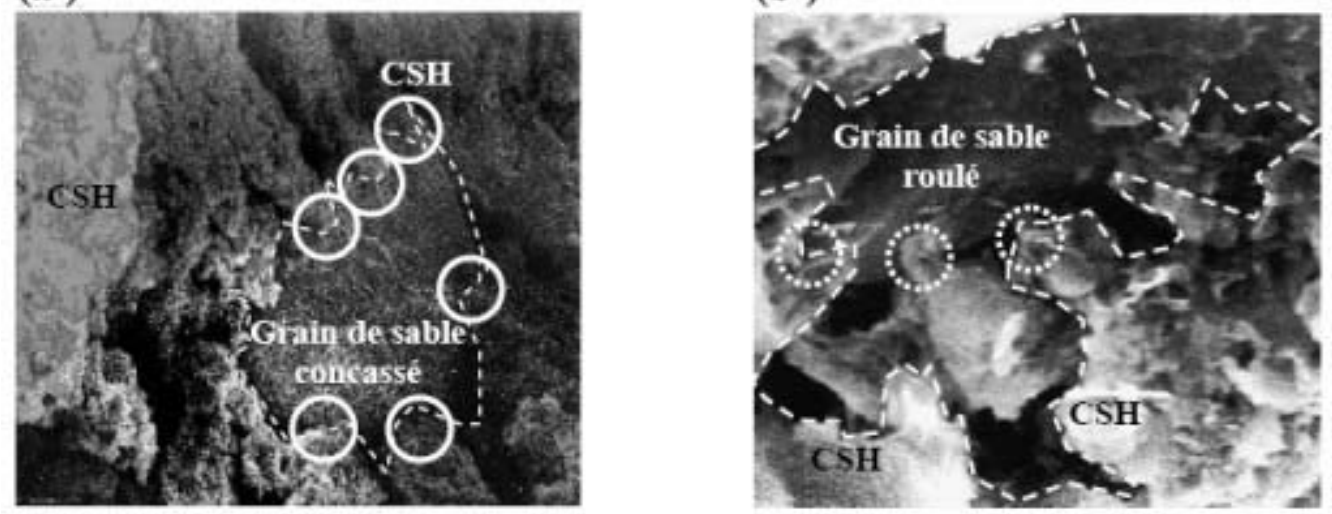

Figure 6. Images en électrons secondaires à 28 jours du sédiment traité avec $10 \%$ de ciment avec $45 \%$ de sable correcteur : (a) et (a') sable concassé, (b) et (b') sable roulé.

\section{Conclusions et perspectives}

Le sable correcteur participe au renforcement de la structure et améliore les performances mécaniques du matériau solidifié. En effet sa présence permet de doubler 
les résistances à la compression simple et d'améliorer les résistances à la compression diamétrale de $10 \%$, quels que soient sa nature granulométrique et son dosage. Les formulations les plus performantes sont constituées avec $45 \%$ de correcteur.

Il existe une répartition granulométrique optimale du correcteur employé permettant de renforcer de manière efficace le squelette granulaire. Il semble qu'un granulat à $\mathrm{C}_{U}$ élevé, bien gradué, apporte de meilleures résistances.

A 28 jours, dans des conditions de cure normale, l'analyse granulométrique des matériaux solidifiés intégrant $45 \%$ de sable correcteur montre une répartition plus grossière sans distinction du type de sable. L'analyse microstructurale au MEB vient confirmer cette tendance en révélant la présence de $\mathrm{CSH}$ autour des particules constituant le matériau.

La nature du sable correcteur, sa forme ainsi que sa distribution granulométrique sont des facteurs d’influence du développement des performances mécaniques du matériau solidifié qu'il convient de prendre en compte.

Afin de diminuer les fractions perturbatrices (M.O. et argiles en particulier) et leurs effets et d'optimiser les performances mécaniques, il pourrait être intéressant de procéder à l'incorporation de correcteurs granulométriques moins organiques, plus grossiers et avec une répartition granulométrique plus large.

La présente étude a été menée de façon empirique. Elle se base sur des mesures expérimentales suivies d'observations. Les études futures nécessiteraient le recours à des méthodes de mélange plus scientifiques et plus pertinentes, avec une correction du fuseau granulométrique maîtrisée, répondant à des modèles d'empilement contrôlés.

La correction peut alors être menée via l'utilisation de matériaux nobles ou de matériaux considérés actuellement comme des déchets dans un objectif de double valorisation.

\section{Références bibliographiques}

BOUTOUIL M. (1998). Traitement de vases de dragage par solidification/stabilisation à base de ciment et additifs. Thèse de l'Université du Havre. 279 p.

BOUTOUIL M., LEVACHER D. (2000). Traitement et valorisation des vases de dragage par solidification/stabilisation : état de l'art. VI ${ }^{\text {èmes }}$ Journées Nationales Génie Côtier - Génie Civil, Caen, pp 367-375. doi:10.5150/jngcgc.2000.031-B

BOUTOUIL M., LEVACHER D. (2001). Étude expérimentale de la solidification d'une vase de dragage à base de ciments : évaluation de la porosité et de la structure poreuse après solidification. Materials and structures, vol. 34, n 3, pp 186-192. doi:10.1007/BF02480510

COLIN D. (2003). Valorisation de sédiments fins de dragage en technique routière. Thèse de l’Université de Caen-Basse-Normandie, 181 p. 
Influence de l'ajout d'un correcteur granulométrique sur les propriétés des sédiments traités aux liants hydrauliques : 8.11

DAMIDOT D., ABRAIAK N.E., DUBOIS V., ZENTAR R., MAC FARLANE F., BULTEEL D., JUNQUA G., KADA H., KAMALI S., REFIF H., WIRQUIN E., GREGOIRE P. (2006). Guide technique régional relatif à la méthodologie de gestion des sédiments de dragage portuaire - Plan régional d'élimination des déchets industriels spéciaux (PREDIS) Nord-Pas-de-Calais. $422 \mathrm{p}$.

DUAN Z. (2008). Caractérisation, stabilisation et solidification de sédiments marins. Thèse de l'Université de Caen-Basse-Normandie, 198 p.

DUBOIS V. (2006). Etude du comportement physico-mécanique et caractérisation environnementale des sédiments marins. Thèse de l'Université d'Artois. 285 p.

GROSDEMANGE D., LEVEQUE F., DROUSIE J.L., AQUA J.L., MEHU J., BAZIN

C. (2008). The SEDIMARD project: Presentation and results. International Symposium on Sediment Management, Lille 9-11 juillet 2008, pp 181-186.

LEVACHER D., COLIN D., PERRONI A.C., DUAN Z., SUN L. (2006). Recyclage et valorisation des sédiments fins de dragage à usage de matériaux routiers. IX ${ }^{\text {èmes }}$ Journées Nationales Génie Côtier - Génie Civil, Brest, pp 603-612. doi:10.5150/jngcgc.2006.058-L

REKIK B. (2007). Propriétés géotechniques des sédiments de dragage traités au ciment. Thèse de l'Université de Caen Basse-Normandie. 122 p.

REKIK B., BOUTOUIL M. (2009). Geotechnical properties of dredged marine sediments treated at high water/cement ratio. Geo-Marine Letters, ${ }^{\circ}$ 29, pp 171-179. doi:10.1007/s00367-009-0134-x

REY F. (1999). Etude physico-mécanique et environnementale du composite vasechaux et additifs. Thèse de l'Université de Caen Basse-Normandie. 271 p.

SANNIER L. (2008). Limites et performances des caractéristiques mécaniques et environnementales de sédiments marins contaminés traités par liant hydraulique Application au pilote SEDI.MAR.D. 83, Rapport Master 2 Génie Côtier, Université de Caen-Basse-Normandie, $106 \mathrm{p}$.

SETRA-LCPC (2000). Traitement des sols à la chaux et/ou aux liants hydrauliques Application à la réalisation des remblais et des couches de forme - Guide technique (GTS). $240 \mathrm{p}$. 
8.12 : Revue Paralia - Vol. 4 (2011) 